\title{
Ultrasonographic Aspect of Coelomic Organs in Boid Snakes
}

\author{
Robert Cristian PURDOIU ${ }^{1 *}$, Luci BEL ${ }^{2}$, Radu LĂCĂTUŞ ${ }^{1}$, Ionel PAPUC ${ }^{1}$ \\ ${ }^{1}$ University of Agricultural Sciences and Veterinary Medicine, Faculty of Veterinary Medicine Department \\ of Semiology, Radiology and Medical Imaging, Mănăştur, no. 3-5, Cluj Napoca, Romania; \\ ${ }^{2}$ University of Agricultural Sciences and Veterinary Medicine, Faculty of Veterinary Medicine Department \\ of Anaesthesiology and Propaedeutic., Mănăștur, no. 3-5, Cluj Napoca, Romania. \\ *Corresponding author: robert.purdoiu@usamvcluj.ro
}

Bulletin UASVM Veterinary Medicine 73(1) / 2016,

Print ISSN 1843-5270; Electronic ISSN 1843-5378

DOI:10.15835/buasvmcn-vm: 11962

\begin{abstract}
The unique anatomic features of the ophidian organs have direct consequences on the approaches used and the structures viewed during an ultrasonography examination; these include the organs mobility in the coelomic cavity and the particular aspect of the organs.

Assessment of optimal approaches of ultrasonography in the boid snakes, so to identify the normal ultrasonographyc anatomy of the coelomic organs.

The biological material was represented by 4 boid snake: 3 Boa constrictor (Boa constrictor imperator) and 1 Burmese Python (Python molurus bivittatus); 1 male and 3 female, weighing between 2.5 and $20.7 \mathrm{~kg}$ and measuring from 1 to $3 \mathrm{~m}$. The snakes were each held both in ventral and dorsal recumbency, with no anesthesia necessary, only physical restraining. For the ultrasonography of coelomic organs examination, an ultrasound Mindray DC-6 unit equipped with a 7.5-10 MHz linear was used. Ventral and intercostals approaches were used, evaluation and measurements being assessed in B mode. In some cases Color Doppler was used to visualize the blood flow in the ophidian organs.

B mode ultrasonography shows the heart that could be taken as a mark point for identifying the other coelomic organs. There were put in evidence the intestine, the liver and gall bladder, the kidney and the ovary. Vascularization of the liver, kidney and the heart was evaluated through Doppler.

The ultrasound examination is a non-invasive method that could constitute a useful tool in identifying different pathologies located at the coelomic level.
\end{abstract}

Keywords: coelomic cavity, constrictor snake, ultrasonography

\section{INTRODUCTION}

Two dimensional B-Mode ultrasonography is a useful and well-adapted tool that helps exploring the aspect of organs and soft tissues both in mammalians and reptiles. Unlike radiology, ultrasound examinations permit differentiation of soft tissues structures and help gain insight of the normal aspect or modifications of the organs. Ultrasonography is an ideal method to gain insight on snake health, being known that reptile show few signs that could indicate a pathological condition (Hernandez-Diver, 2008). Medical imaging techniques begin to gain importance among veterinarian that work with reptiles and snakes (Banzato et al., 2013), but also among snake breeders as a tool for pregnancy management (Gnudi et al., 2009, Banzato et al., 2013, Purdoiu et al., 2014). Putting a correct diagnostic base on ultrasonography examination finding require a good knowledge of normal ultrasound anatomy of the coelomic organs in snakes and relies on highresolution equipment (Silverman, 2005; Pees, 2010). 


\section{MATERIALS AND METHODS}

The biological material was represented by 4 boid snake: 3 Boa constrictor (Boa constrictor imperator) and 1 Burmese Python (Python molurus bivittatus); 1 male and 3 female, weighing between 2.5 and $20.7 \mathrm{~kg}$ and measuring from 1 to $3 \mathrm{~m}$.

The snakes were each held both in ventral and dorsal recumbency, with no anesthesia necessary, only physical restraining.

For the ultrasonography of coelomic organs examination, an ultrasound Mindray DC-6 unit equipped with a 7.5-10 MHz linear probe and a GE Logique E9, linear probe 7-15 MHz were used. The choosing the correct transductor is not a problem, especially in large individuals, when a convex transducer can be easily used (Pees, 2011).

For assessing the aspect of coelomic organs 2 ultrasonographic coupling sites were used, a ventral and intercostal approaches evaluation and measurements being assessed in B mode. In some cases Color Doppler was used to visualize the blood flow in the ophidian organs.

The snakes were examined without sedation, depending on the size of the animal, two to four assistants helped to restrain the snakes (Fig. 1). Prior to the examination a thick layer of contact gel was applied on the ventral side and on the lateral side of the snake.

\section{RESULTS AND DISCUSSION}

The heart was located by visualization of movement of the ventral scutes over the heart and by palpation.

Two-dimensional echocardiographic images were obtained by placing the transducer head directly on the ventral midline over the heart or laterally, on the right intercostal space corresponding to the heart (Fig. 2).

Long axis (sagittal) images were obtained by aligning the ultrasound beam with the long axis of the snake on the midline and then angling

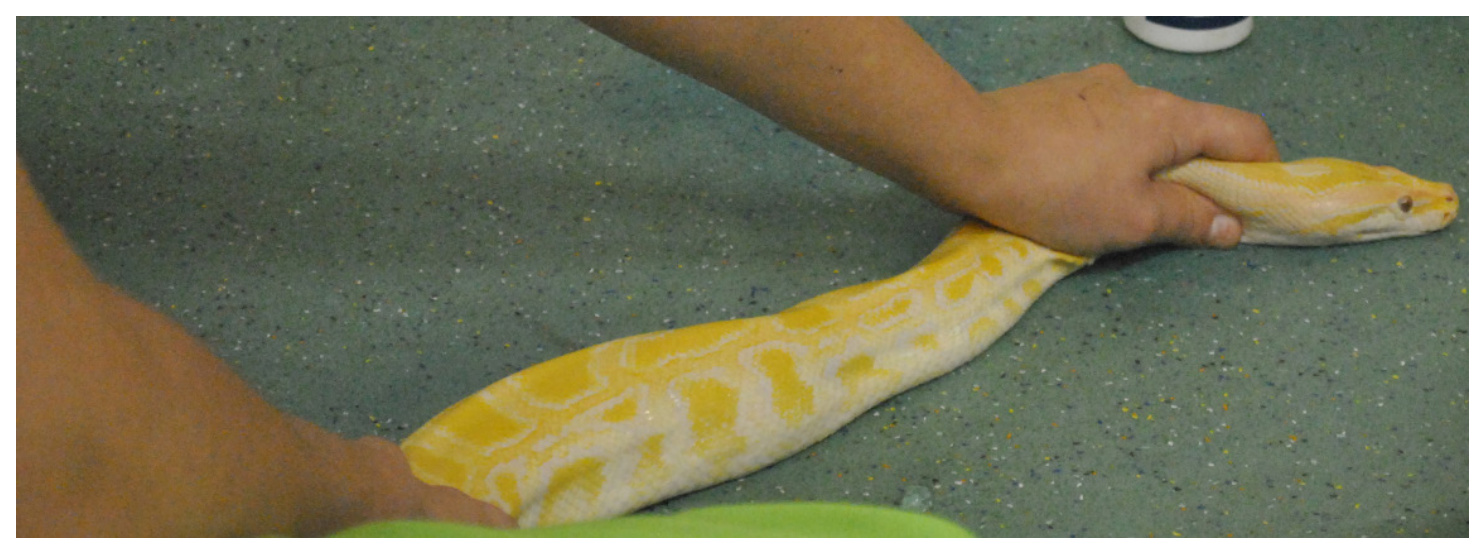

Fig. 1. Restraint in ventral recumbency of an albino P.m.bivittatus

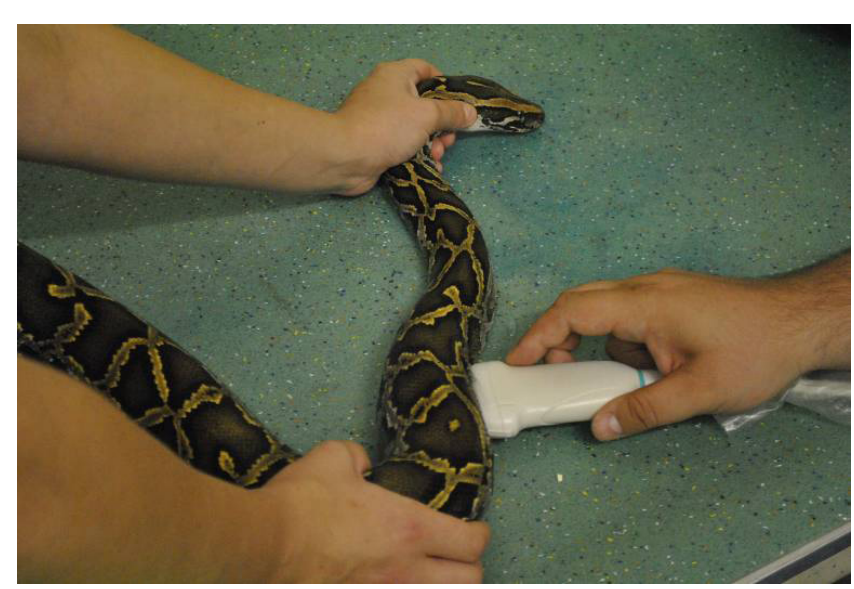

Fig. 2. Probe positioning for heart evaluation

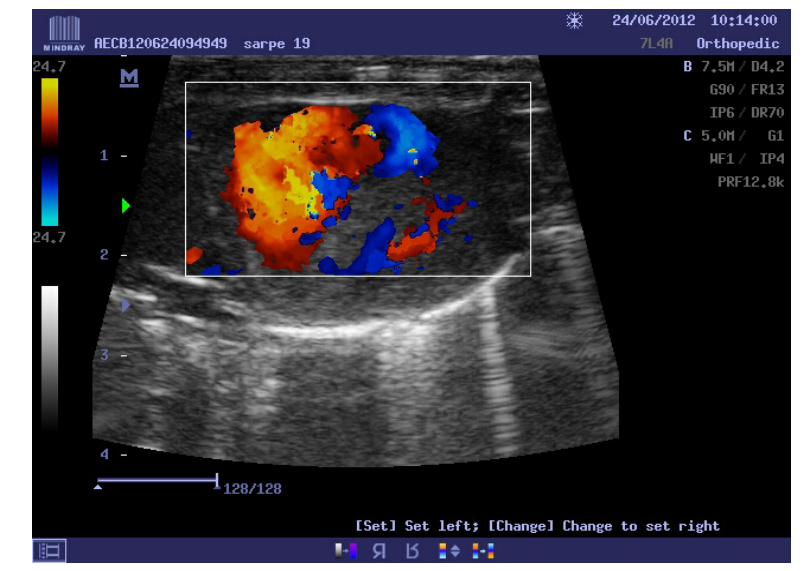

Fig. 3. Doppler ultrasonography of the heart 
the transducer to the right and then left (ventral approach) and smooth lateral movements (intercostal approach). While maintaining the midline position, the transducer head was rotated $90^{\circ}$ from the midsagittal plane and angled cranially from the apex to the base of the heart to obtain the short axis (transverse) images.

Trans-ventricular and subarterial short axis sections were analyzed, assessing the ventricle (Fig. 3) and the origin of the great vessels respectively (Fig. 4-5). The Doppler image of the trans ventricular section shows mixed arterial and venous blood going out during the systole.

The long axis evaluation of the heart show the ventricle with the cavum arteriosum and cavum pulmonare, in this position is also evident the left atrium, the pulmonary trunk, the aortic arch, the ventricle and the left atrium and visualization of the atrio-ventricular valvae and the blood flow from the ventricle to the three vessels (Fig. 5-8).

Short axis view, show most of the anatomical characteristics of the ophidian heart: the ventricle myocardium in both systole and diastole, the two atria, the pulmonary trunk and aortic arches and the sinus venosus (Fig. 6).

Because of the fact that the ophidian heart shows two types of movements, the systolic movement, characteristic in all animals, and also an "up and down" movement, due to the fact that the heart is not suspended by any ligament in the body, we can presume that no measurements in B mode are exact, but purely informative (Bel et al., 2012).

In snake, the liver is elongated and situated caudally to the heart (Schilder etal.,1994). Between the heart and liver the lungs are interposed, they decrease in size toward the anterior extremity of body and liver size increases as the transducer is moved to the middle third of the body (Fig. 9). By Doppler ultrasound in the liver may be visible the central hepatic vein which makes it easy to distinguish the liver from the body fat, adipose formation located on the rear underside of the body.

Bile ducts have echogenic appearance being visible in the liver parenchyma of the large specimens. In small specimens the liver looks smooth, biliary ducts being hardly noticeable.

Gall bladder is easily identifiable in individuals of size below $1.5 \mathrm{~m}$; in large individuals it is difficult to identify the gall bladder due to its mobility. Large individuals are hardly restrained because body muscles are very well developed, arced continuously, which causes the gall bladder to change its position. Gall bladder shape is usually spherical, sometimes can have a pear aspect.

Gall bladder has anechoic aspect being located at the caudal pole of the liver (Fig. 10-12). The gall bladder can be evidenced both by intercostal and

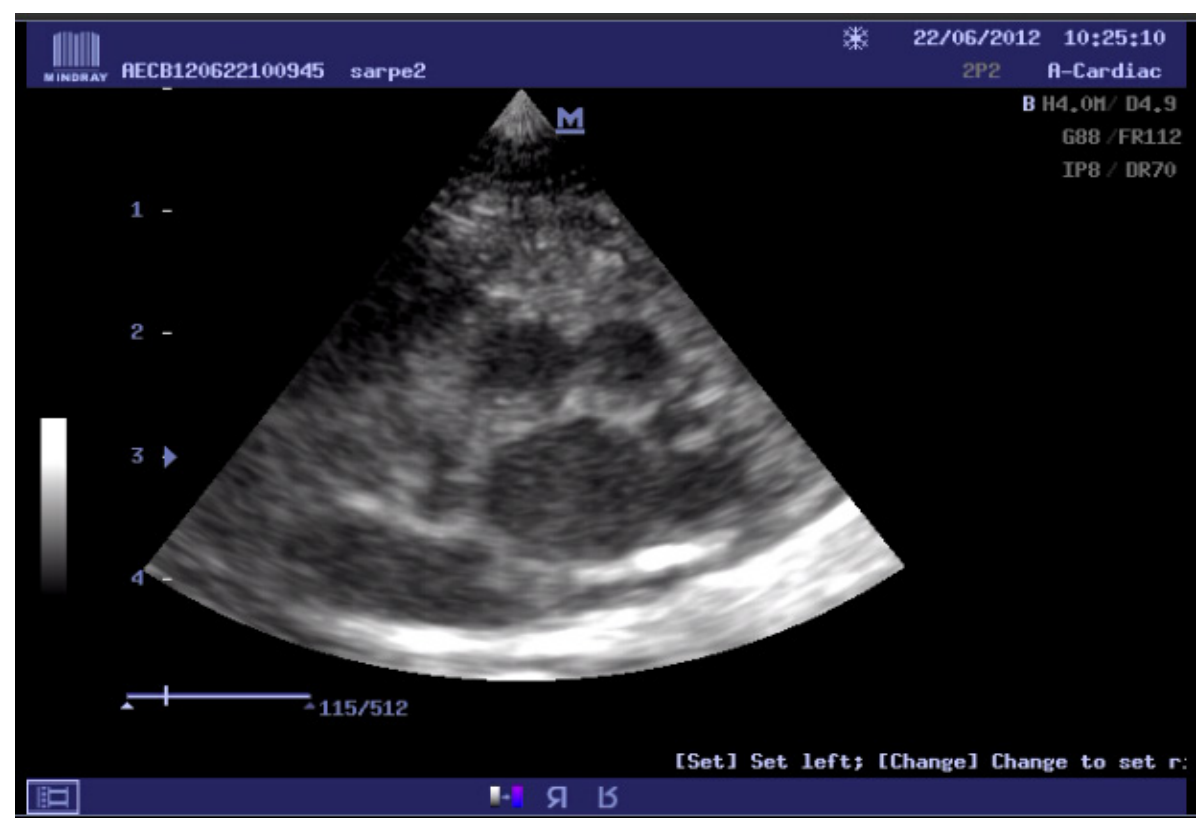

Fig. 4. Trans ventricular, subarterial short axis 
ventral approach. The same is true for the liver parenchyma.

The spleen is spherical shape, small size, with increased echogenicity compared with the liver (Mannion, 2006) and it is difficult to identify ultrasound, is visible at large specimens.

The stomach is elongated, being difficult to identify ultrasonographicaly and is visible only when it is distended by fluid or gas.

The pancreas usually cannot be identified by ultrasound, in terms of echogenicity it is less echogenic than the liver and spleen (Mannion, 2006)

The small intestine is long, and to be able to distinguish between the intestinal tract and blood vessels, Doppler technique can be used to reveal blood flow. The B mode ultrasound identifies the intestine by highlighting the motion content (Fig. 13).

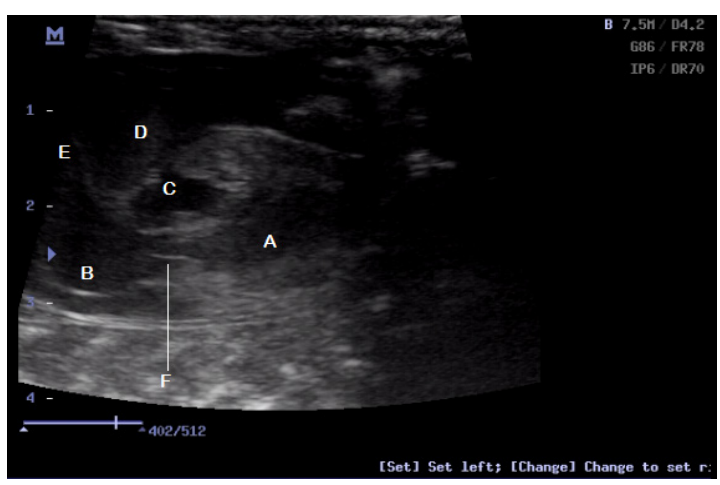

Fig. 5. Long axis view: A. Cavum venosum and cavum arteriosum; B. Left atrium; C. Cavum pulmonare;

D. Aortic arch; E. Pulmonary trunk; F. Atrio- ventricular valvae

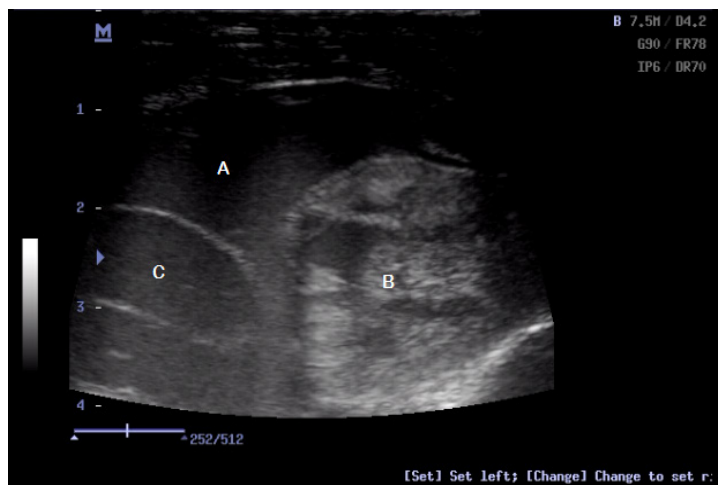

Fig. 7. Long axis intercostal view: A. Left atrium; B. Ventricle; C. Pulmonary trunk and aortae
The kidneys are paired organs located in the third caudal part of the body. Right kidney is placed cranial to the left kidney and is situated on both sides of the spine. Quality ultrasound images are obtained regardless of the approach (intercostal or ventral - Fig. 14-15).

Highlighting the renal vasculature can be done by using ultrasound Doppler mode, which can identified venous collaterals (Fig. 16).

The $\mathrm{B}$ mode ultrasound is a valuable technique for assessing the pregnancy in snake (Stetter, 2006, Purdoiu et al., 2014), helping the vet to identify the stage of pregnancy base on the aspect of the eggs (fig. 17).

\section{CONCLUSION}

The ultrasound is a non-invasive diagnostic method and can be used for identifying the aspect of the coelomic organs in snake, being

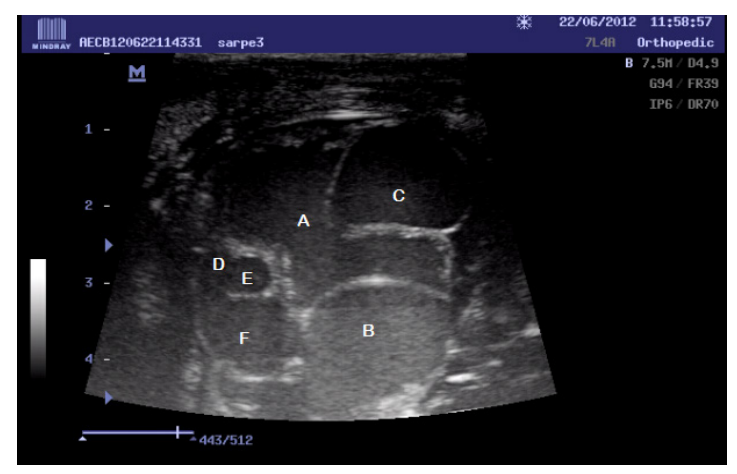

Fig. 6. Short axis intercostals view of A. Right atrium; B. Left atrium; C. Sinus venosus; D, E aorta; F. pulmonary trunk

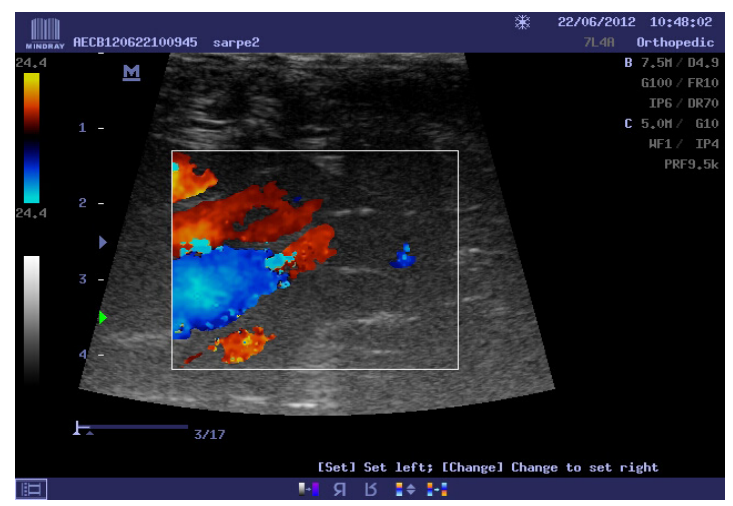

Fig. 8. Doppler image of blood flow through the Pulmonary trunk ant the two aortic arches 


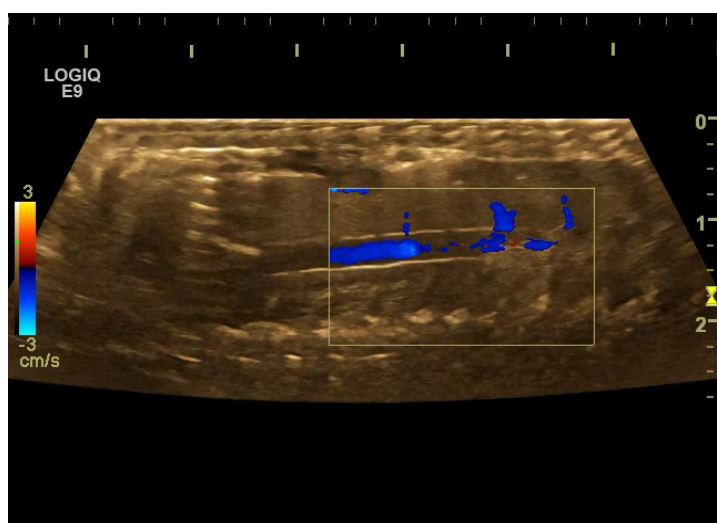

Fig. 9. The snake liver, intercostal approach, highlighting the central hepatic vein $11 \mathrm{MHz}$

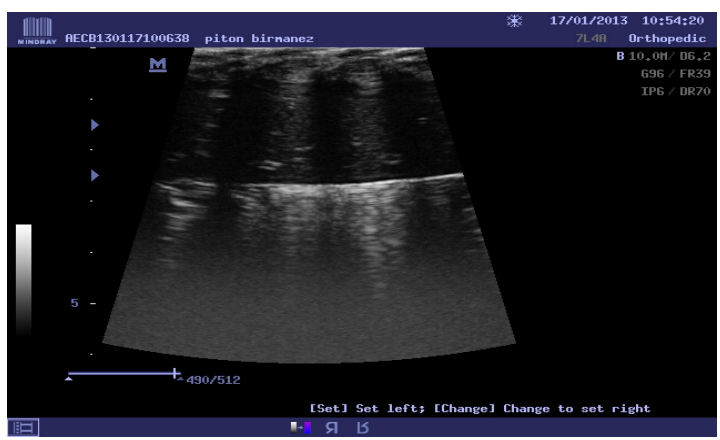

Fig. 11. Snake liver, bile ducts highlighting, $10 \mathrm{MHz}$

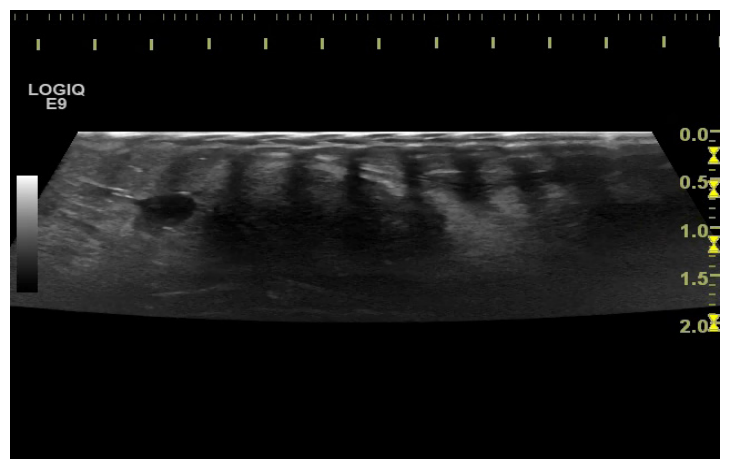

Fig. 10. The snake gallbladder, anechoic aspect 11 $\mathrm{MHz}$

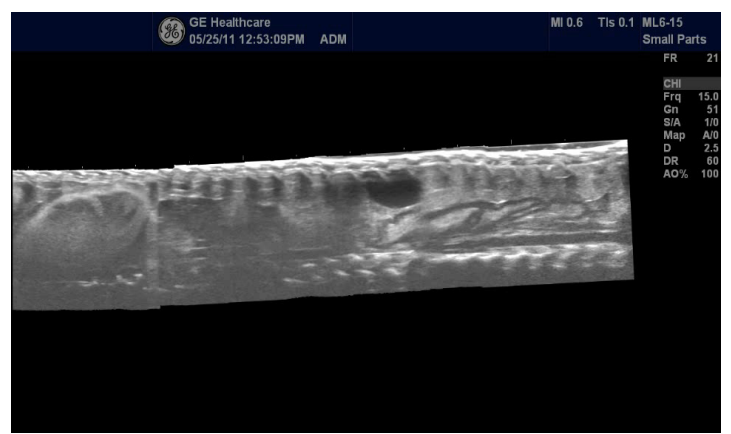

Fig. 12. Liver (white arrow) and gallbladder (blue arrow), intercostal approach, panorama, $15 \mathrm{MHz}$

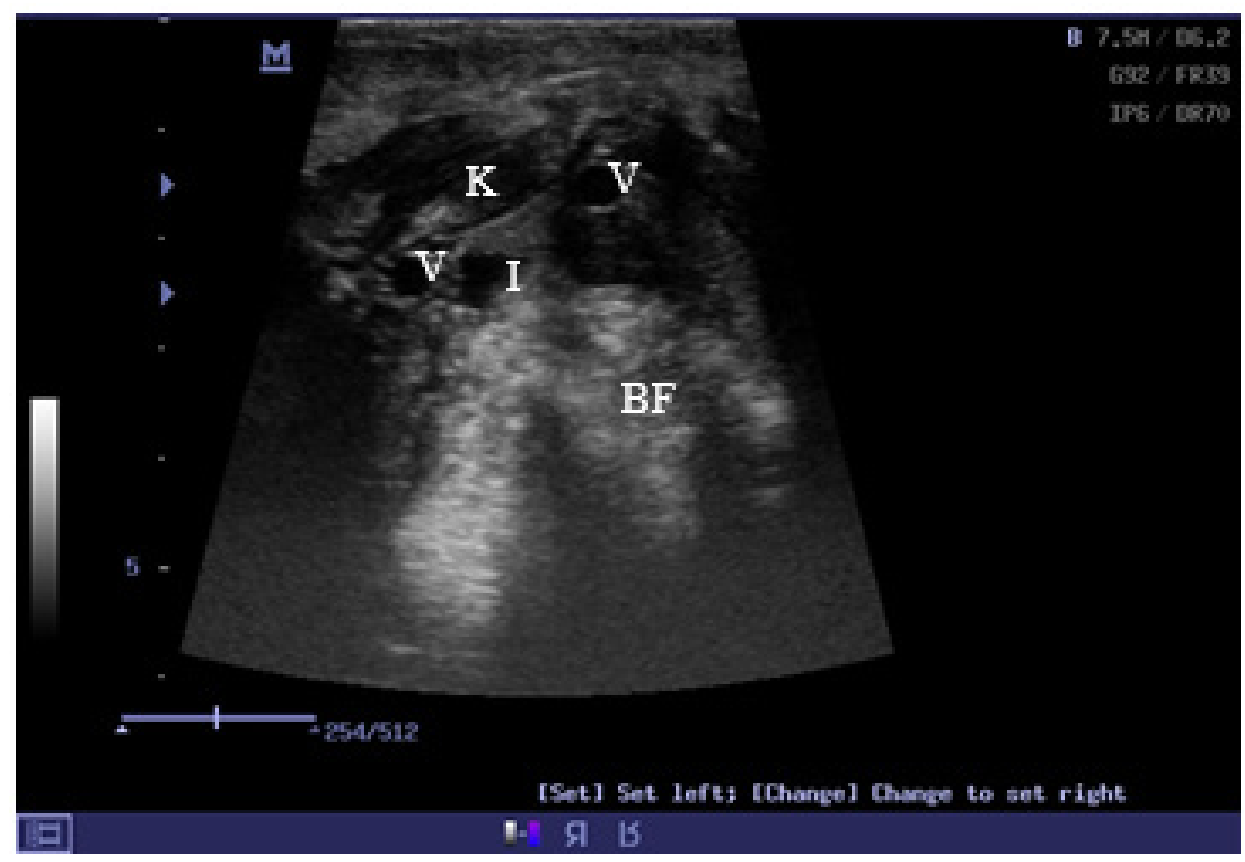

Fig. 13. Transverse approaches: I - small intestine, $\mathrm{K}$ - kidney, V - blood vessels, BF - body fat; $7.5 \mathrm{MHz}$ 


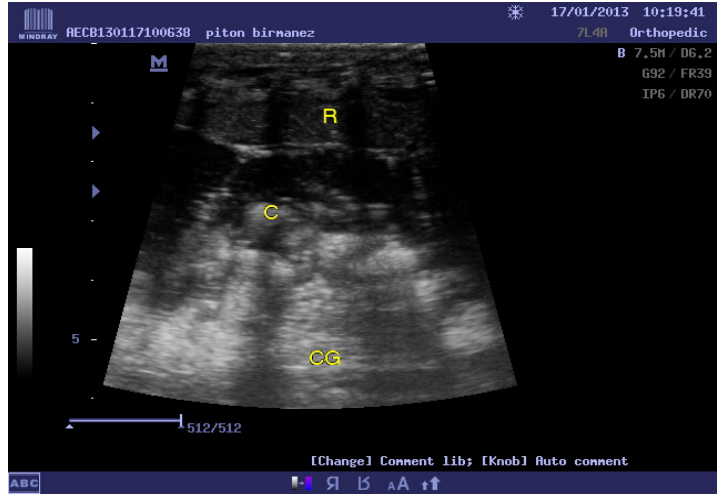

Fig. 14. Sagittal approaches: R - kidney, C intestine, CG - body fat; $7.5 \mathrm{MHz}$

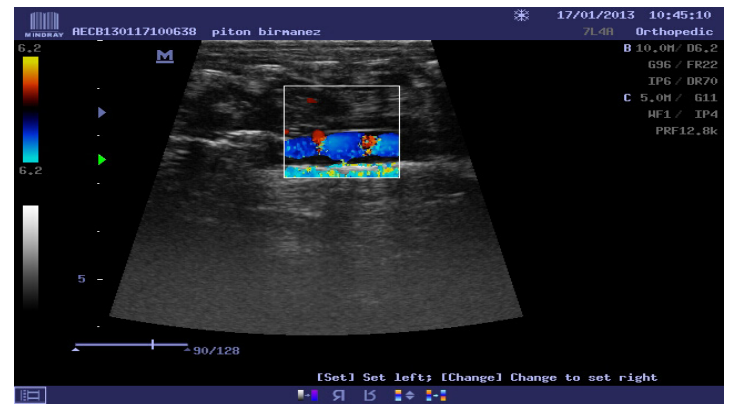

Fig. 16. Doppler ultrasound - highlighting the renal veins, $10 \mathrm{MHz}$

well tolerated by the animal without inducing stress. The best images were obtained using linear transducers with frequency between 7.5-15 MHz. The ultrasonographic approach can be done intercostal or on the ventral side of the body. The $B$ mode ultrasound help assess the aspect of the coelomic organs and this method can be used as a routine examination tool.

\section{REFERENCES}

1. Banzato T, Hellebuyck T, Van Caelenberg A, Saunders JH, Zotti A (2013). A review of diagnostic imaging of snakes and lizards. Veterinary Record 173:43-49.

2. Bel L, Purdoiu RC, Peștean C, Ober C, Oana L, Mircean $M$ (2012). Aspects of B mode echocardiography of the Burmese Python (Python molurus bivittatus). Bulletin UASMV, Veterinary Medicine 69(1-2): 218-219.

3. Gnudi G, Volta A, Di Ianni F, Bonazzi M, Manfredi S, Bertoni G (2009). Use of ultrasonography and contrast radiography for snake gender determination. Veterinary Radiology \& Ultrasound 50:309-311.

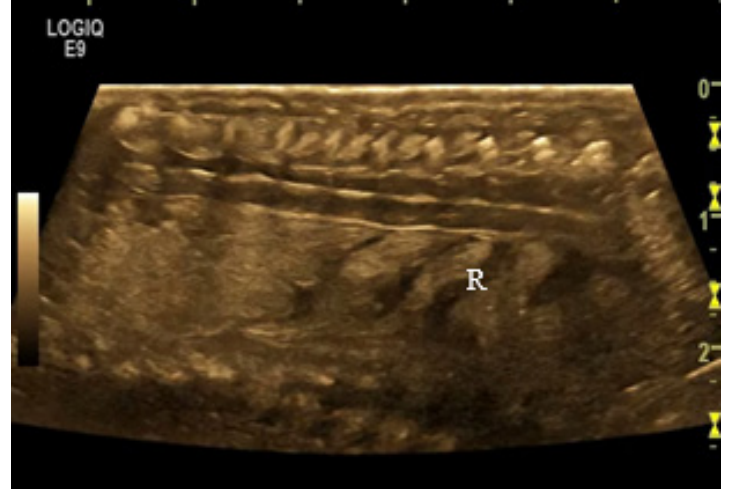

Fig. 15. Sagittal approaches: R - kidney aspect 11 $\mathrm{MHz}$

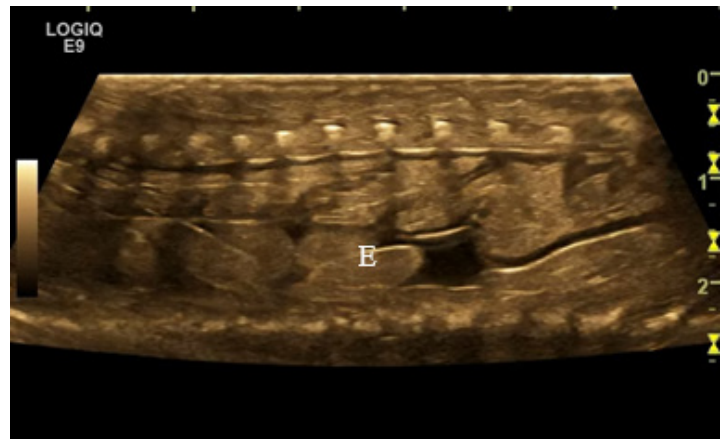

Fig. 17. Eggs development in snake, $10 \mathrm{MHz}$

4. Hernandez-Divers SJ (2008). Snake clinical procedures and diagnostics. NAVC Conference Exotic - Reptiles, 17521756.

5. Mannion $P$ (2006). Diagnostic ultrasound in small animale practices, Blackwell Sciences.

6. Pees M (2010), Ultrasonography. In: KrautwaldJunghanns ME, Pees M, Reese S, Tully T(eds). Diagnostic Imaging of Exotic Pets: Birds, Small Mammals, Reptiles, Schluetersche Verlagsgesellschaft mbH \& Co KG, 334-356.

7. Pees M (2011), Ultrasonography. In : KrautwaldJunghanns ME, Pees M, Reese S, Tully T(eds). Diagnostic Imaging of Exotic Pets: Birds, Small Mammals, Reptiles, Schluetersche Verlagsgesellschaft mbH \& Co KG, 334-336.

8. Purdoiu RC, Lăcătuş R, Papuc I (2014). Ultrasonographic aspects during pregnancy in snakes. Lucrări Ştiinţifice USAMV Iași - seria Medicină Veterinară 57(1-2):6-12.

9. Schildger B, Casares M, Kramer M et al (1994). Technique of ultrasonography in lizards, snakes, and chelonians. Semin Amian Exot Pet Med 3:147-155.

10. Silverman S (2005). Diagnostic imaging. In : DR Mader (ed). Reptile Medicine and Surgery, 2nd ed, Saunders, 471-489.

11. Stetter MD (2006). Ultrasonography. In : Mader DR (ed). Reptile Medicine and Surgery, Saunders. 